\title{
A STOCHASTIC CHARACTERIZATION OF HARMONIC MORPHISMS
}

\section{Laszlo Csink and Bernt $\varnothing$ ksendal}

\section{Abstract}

It is proved that the harmonic morphisms between two $\rho$-harmonic spaces $(X, U)$ and $(Y, \mho)$ are exactly the continuous functions $\phi$ : $\mathfrak{X} \rightarrow \mathscr{Y}$ which map a Ray process $z_{t}$ whose excessive functions are $U$-hyperharmonic into the diffusion $Y_{t}$ associated to $(y, v)$. The branch set $B$ of $Z_{t}$ is identified as the set of points where $\phi$ is finely locally constant. We also obtain that for any $x \in \mathcal{X}$ either $\phi$ is finely locally constant at $x$ or $\phi$ maps every fine neighbourhood of $x$ onto a fine neighbourhood of $\phi(x)$.

If $B=\varnothing$, i.e. $\phi$ is finely locally non-constant, then $z_{t}$ can be replaced by a continuous time change of the diffusion $x_{t}$ associated to $(X, U)$.

This allows one to use stochastic methods in the investigation of harmonic morphisms. For example, as an application we prove that if $H$ is a polar set in $y$, then $\phi^{-1}(H) \wedge B$ is a polar set in $X$. We also obtain a general boundary value result for harmonic morphisms. 

A STOCHASTIC CHARACTERIZATION OF HARMONIC MORPHISMS

Laszlo Csink and Bernt $\varnothing$ ksendal

\section{§1. Introduction}

The main purpose of this paper is to identify the harmonic morphisms between two $\rho$-harmonic spaces as the path-preserving functions of certain associated Markov processes. This opens for the use of stochastic methods in the investigation of harmonic morphisms and we give some examples of applications.

Before we formulate the results more precisely, we recall briefly some basic results about the connection between $\mathcal{\rho}$-harmonic spaces and their associated Markov processes:

Let $(\mathcal{X}, \mathcal{U})$ be a $\mathcal{P}$-harmonic space in the sense of Constantinescu \& Cornea [7]. ( $X$ is a locally compact Hausdorff space with a countable base, $u$ a hyper-harmonic sheaf.) Assume that $\mathcal{X}$ is connected and that 1 is superharmonic. Let $p>0$ be a bounded, strict, continuous potential on $\nVdash$. Let * denote the specific multiplication (defined in [7], p. 189) wrt. the abstract carrier Sp $=X-U$, where $U$ is the maximal open set on which $p$ is harmonic. According to Theorem 10.2 .1 p. 252 in [7] there exists a (unique) sub-Markov semigroup $P_{t^{\prime}} t \geqslant 0$ such that

$$
(f \star p)(x)=\int_{0}^{\infty}\left(P_{t} f\right)(x) d t
$$

for all $t \geqslant 0, x \in \mathcal{X}$ all $f \in C_{C}^{+}(x)$, where $C_{C}^{+}(x)$ denotes the non-negative continuous functions on $¥$ with compact support. 
We also obtain (see Theorem 10.2 .2 in [7])

$$
\begin{aligned}
& \lim _{t \downarrow 0} \frac{1}{t}\left(P_{t} f\right)(x) \rightarrow 0 \text { uniformly in } x \text { on compacts } \\
& \text { outside } \operatorname{supp}(f) \text {, for all } f \in C_{C}^{+}(x)
\end{aligned}
$$

and

$$
P_{t}\left(C_{0}\right) \subset C_{0}
$$

where $C_{0}$ denotes the set of continuous functions on $\mathcal{X}$ which vanish at $\infty$. Therefore by Theorem 9.4, p. 46 and Prop. 9.10, p. 50 in Blumenthal \& Getoor [2] there exists a standard process $\left\{x_{t}\right\}_{t \geqslant 0}$ with continuous paths (i.e. a diffusion) having $P_{t}$ as its transition semigroup. (See [2], p. 45 for definition of standard processes.) We shall call $x_{t}$ the process associated to the $\mathcal{P}$-harmonic space $(x, u)$ and the potential p. A (real) continuous function $h$ on an open set $U \subset x$ will then be $(\mathfrak{X}, U)-$ harmonic if and only if

$$
\mathrm{h}(\mathrm{x})=\mathrm{E}^{\mathrm{x}}\left[\mathrm{h}\left(\mathrm{x}_{\tau_{\mathrm{V}}}\right) \cdot \chi_{\left\{\tau_{\mathrm{V}}<\infty\right\}}\right] ; \mathrm{x} \in \mathrm{V}
$$

for all relatively compact open sets $V$ with $\bar{V} \subset U$, where $\tau_{V}=$ $\inf \left\{t>0 ; x_{t} \notin V\right\}$ is the first exit time of $x_{t}$ from $V$ and $E^{x}$ denotes the expectation wrt. the probability law $\mathrm{P}^{\mathrm{x}}$ of $\mathrm{x}_{t}$ starting at $x$. (See 12.18. C p.24 in Dynkin [9].) The Green measure (wrt. $x_{t}$ ), $G^{U}(\cdot, \cdot)$, is defined by

$$
G^{U}(x, F)=E^{x}\left[\int_{0}^{\tau_{U}} \chi_{F}\left(x_{t}\right) d t\right] \text { for } x \in U, F \subset U \text {. }
$$

In other words,

$$
G^{U}(x, f)=E^{x}\left[\int_{0}^{\tau} f\left(x_{t}\right) d t\right], \quad f \in C_{C}^{+}(U)
$$


Combining (1.1) with (1.7) we conclude that if $F \subset U$ is compact and $f \in C_{C}^{+}(U), f=1$ on $F$ then

$$
\begin{aligned}
G^{U}(x, F) & =E^{x^{x}}\left[\int_{0}^{\infty} \chi_{F}\left(x_{t}\right) \chi_{\left\{t<\tau_{U}\right\}} d t\right] \leqslant \int_{0}^{\infty} E^{x}\left[f\left(x_{t}\right)\right] d t \\
& =\int_{0}^{\infty}(P t f)(x) d t=(f \star p)(x)<\infty
\end{aligned}
$$

Thus the expected length of time this process $x_{t}$ stays in $F$ before exiting from $\mathrm{U}$ is finite. In particular:

The exit time from a compact set is finite a.s. for the process $\mathrm{x}_{t}$.

Therefore (1.4) may be written

$$
h(x)=E^{x}\left[h\left(x_{\tau_{V}}\right)\right] \quad ; x \in V
$$

for all relatively compact $\mathrm{V}, \overline{\mathrm{V}} \subset \mathrm{U}, \mathrm{h}$ harmonic in $\mathrm{U}$.

Let $(x, u)$ and $(y, \vartheta)$ be $\rho$-harmonic spaces. A continuous function $\phi: \mathscr{X} \rightarrow \mathscr{Y}$ is called a harmonic morphism if for any open set $\mathrm{V} \subset \mathrm{Y}$ and any hyperharmonic function $\mathrm{h}$ on $\mathrm{V}$ we have that ho $\phi$ is hyperharmonic on $\phi^{-1}(\mathrm{~V})$. (See e.g. Constantinescu \& Cornea [6], Fuglede [10] and Laine [15].)

Next we explain what we mean by path-preserving functions:

Let $\left(\mathrm{X}_{t}, \Omega, \mathrm{P}^{\mathrm{X}}\right)$ and $\left(\mathrm{Y}_{t}, \hat{\Omega}, \hat{\mathrm{P}}^{\mathrm{Y}}\right)$ be two path-continuous standard processes with locally compact state spaces $\mathfrak{X}, \mathcal{Y}$ and probability laws $\mathrm{P}^{\mathrm{X}}, \hat{\mathrm{P}}^{\mathrm{Y}}$ respectively, $\mathrm{x} \in \mathfrak{X}, \mathrm{y} \in \mathcal{Y}$. A continuous function $\phi: X \rightarrow Y$ is called $\underline{X}_{t} \underline{-Y}_{t}$ path-preserving if for all $x \in \mathfrak{X}$ there exists a relatively compact neighbourhood $D_{3 x}$ and a time change $\alpha_{t}=\inf \left\{s ; \beta_{s}>t\right\}$, where $\beta_{t}(\omega)$ is a continuous non-decreasing additive functional $x_{t}$, such that -up to the exit time $\tau_{D}$ from $D$-the process $\phi\left(x_{\alpha_{t}}\right)$ 
coincide in law with (i.e. has the same finite-dimensional distributions as) $Y_{t}$. The time change is called continuous if $\alpha_{t}$ is continuous. We now explain this more precisely: Let the $\beta_{\tau_{D}}$ - welding of $\phi\left(\mathrm{X}_{\alpha}\right)$ and $\mathrm{Y}_{t}$ be the process

$M_{t}: \Omega \times \hat{\Omega} \rightarrow \quad$ defined by

$$
M_{t}(\omega, \hat{\omega})= \begin{cases}\phi\left(X_{\alpha_{t}}\right) ; & t \leqslant \beta_{\tau_{D}} \\ Y_{t-\beta}{ }_{\tau_{D}}, & t \geqslant \beta_{\tau},(\omega, \hat{\omega}) \in \Omega \times \hat{\Omega},\end{cases}
$$

with probability law $R^{Y}$ given by (with $0 \leqslant t_{1}<\ldots<t_{n}$ )

$$
\begin{aligned}
& R^{Y}\left[M_{t_{1}} \in E_{1}, \ldots, M_{t_{n}} \in E_{n}, t_{k} \leqslant \dot{\beta}_{\tau_{D}}<t_{k+1}\right]=
\end{aligned}
$$

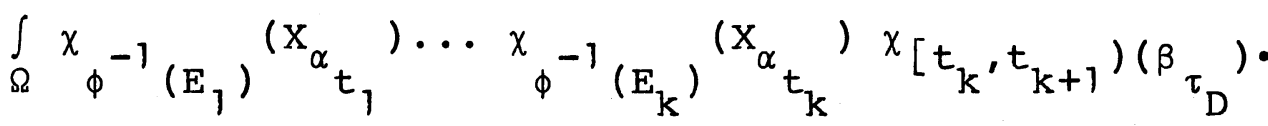

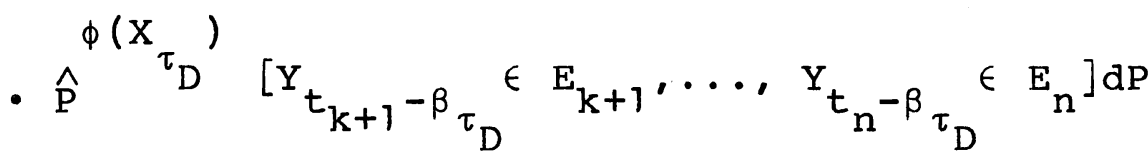

where $\mathbf{x}=\psi(y), \psi: \phi(\bar{D}) \rightarrow \bar{D}$ is a measurable right inverse of $\phi$. (See Csink \& Øksendal [8], pp. 221-222.) Then by saying that $\phi$ is $\mathrm{x}_{t}-\mathrm{Y}_{t}$ path-preserving we mean that for all $\mathrm{x} \in \mathcal{X}$ there exists a neighbourhood $D=\mathbf{x}$ and an additive functional $\beta_{t}$ such that the $\beta_{\tau_{D}}$-welding of $\phi\left(\mathrm{X}_{\alpha_{t}}\right)$ and $\mathrm{Y}_{t}$ coincides in law with $\mathrm{Y}_{t^{\prime}}$ for all right inverses $\psi$ of $\phi$.

The connection between harmonic morphisms in the classical case (where $h$ harmonic means $\Delta h=0$ ) and Brownian path-preserving function goes back to $P$. Levy in the case where $x=y=\mathbb{R}^{2}$ (see McKean [16], where a proof is given using stochastic integrals) and was extended to arbitrary dimensions by Bernard, Campbell \& Davie [1]. Considering the fine topology on $\mathbb{R}^{n}$ the equivalence of finely (classical-) harmonic morphisms and Brownian path-preserving functi ons was proved by $\varnothing \mathrm{ksendal}$ [17]. Csink \& $\varnothing \mathrm{ksendal}$ [8] established the 
identity between harmonic morphisms $\phi: X+\mathscr{Y}$ and $x_{t}-Y_{t}$ pathpreserving functions in the case where the processes $X_{t^{\prime}} Y_{t}$ associated to the harmonic spaces $(x, u)$ and $(y, v)$ are diffusions, i.e. path-continuous standard processes on Euclidean spaces such that the domain of their characteristic operators include the $\mathrm{C}^{2}$-functions. In this article we establish a similar identity in general, using a quite different method. More precisely, we prove that a continuous function $\phi: X \rightarrow Y$ is a harmonic morphism if and only if there exists a Ray process $z_{t}$ on $\mathfrak{X}$ with the property that all the $z_{t^{-}}$ excessive functions are hyperharmonic and such that $\phi$ is $\mathrm{Z}_{t}-\mathrm{Y}_{\mathrm{t}}$ pathpreserving without time change, i.e. $\phi\left(z_{t}\right)$ has the same law as $Y_{t}$. (Theorem 1) (See [13] for information about Ray processes). Then we prove that the branch set of $z_{t}$ can be characterized as the set of points where $\phi$ is finely locally constant. (Theorem 2)

This leads to the following complete description of when a harmonic morphism $\phi$ is finely open:

For each $\mathrm{x} \in \mathfrak{X}$ either $\phi$ is finely locally constant at $\mathrm{x}$ or else $\phi$ maps every fine neighbourhood of $x$ onto a fine neighbourhood of $\phi(x)$. (Corollary 1 ). If $B=\phi$, i.e. if $\phi$ is finely locally nonconstant, then $z_{t}$ can be replaced by a continuous time change of $x_{t}$. As an application we prove that if $H$ is a polar set in $y$, then $\phi^{-1}(\mathrm{H}) \backslash \mathrm{B}$ is a polar set in $X$. We also obtain a general boundary value result for harmonic morphisms.

\section{§2. THE MAIN RESULT}

From now on we will let $\left(\mathrm{X}_{t^{\prime}} \mathrm{P}^{\mathrm{X}}\right),\left(\mathrm{Y}_{t^{\prime}}, \hat{\mathrm{P}}^{\mathrm{Y}}\right)$ be diffusions, i.e. standard processes with continuous paths, associated to the two $\rho$-harmonic spaces $(x, u),(y, v)$. More precisely, we fix two bounded, strict, continuous potentials $p, q$ on $x, y$ and construct $x_{t}, y_{t}$ from $p, q$ as explained in the introduction. 
In the following $\bar{W}$ and $\mathrm{W}^{0}$ denote the closure and the interior respectively, of the set $W, C_{C}(W)$ denotes the continuous functions on $W$ with compact support, $C^{b}(W)$ denotes the bounded continuous functions on $W$. If $\bar{W}$ is compact, $\bar{W} \subset V$ we write $W \subset \subset V$. Otherwise notation will be as in [7]. A Borel set $H \subseteq X$ is called finely open (wrt. $\mathrm{x}_{t}$ ) if $\tau_{\mathrm{H}}>0$ a.s. $\mathrm{P}$, for all $\mathrm{x} \in \mathrm{H}$. A function $\mathrm{f} \geqslant 0$ is called excessive (wrt. $\mathrm{X}_{t}$ ) on a finely open set $\mathrm{H}$ if (see Dynkin [9], Th. 12.2)

$$
\mathrm{E}^{\mathrm{X}}\left[\mathrm{f}\left(\mathrm{X}_{\tau}\right)\right] \leqslant \mathrm{f}(\mathrm{x}) \text { for all stopping times } \tau<\tau_{\mathrm{H}^{\prime}}, \mathrm{x} \in \mathrm{H}
$$

and

$$
\lim _{k \rightarrow \infty} E^{x}\left[f\left(x_{\tau_{k}}\right)\right]=f(x) \text {, for all } x \in H \text {, }
$$

for any sequence $\left\{\tau_{k}\right\}$ of stopping times such that $\tau_{k} \rightarrow 0$ a.s. $P^{X}$ as $k \rightarrow \infty$. The fine topology on $\mathfrak{X}$ in the potential theoretic sense is identical with the $x_{t}$-fine topology, and similarly for $y$ and $Y_{t} \cdot$

We say that a locally bounded function $f$ is finely harmonic (wrt. $x_{t}$ ) in a finely open set $H$ if for any relatively compact, finely open set $\mathrm{V} \subset \mathrm{H}$ on which $f$ is bounded we have that $f$ and $M-f$ are excessive in $V$, where $M=\sup \{f(x) ; x \in V\}$.

We refer to Bliedtner and Hansen [4] for more information about the stochastic representation of the various potential theoretic notions. For example, the balayage $\widehat{R}_{f}^{A}$ of a non-negative hyperharmonic function $f$ is given by

$$
\left(\hat{R}_{f}^{A}\right)(x)=E^{x}\left[f\left(X_{T_{A}}\right)\right]
$$

where $T_{A}=\inf \left\{t>0 ; x_{t} \in A\right\}$ is the first hitting time of $A([4]$, Theorem VI. 3.14). 
In order to establish our main result we need to extend the construction in [7] described in the introduction: We will show how to associate a stochastic process to any positive continuous superharmonic function, not just to a bounded continuous strict potential. The resulting process will not be a diffusion in general, but it turns out that it is always a Ray process. This is a stochastic process $z_{t}$ which is corlol (continuous from the right, with left limits) with life time $\xi, 0 \leqslant \xi \leqslant \infty$, and whose resolvent

$$
\left(U_{\alpha} f\right)(x)=E^{x}\left[\int_{0}^{\xi} e^{-\alpha t} f\left(z_{t}\right) d t\right] \quad ; \alpha>0
$$

is a Ray resolvent in the sense of $[13]$, i.e.

(i) $\quad \alpha U_{\alpha}^{1} \leqslant 1$ for $\alpha>0$

(ii) $U_{\alpha}=U_{\beta}+(\beta-\alpha) U_{\alpha} U_{\beta}$ for $\alpha, \beta>0$.

(iii) $U_{\alpha} f \in C_{0}(x)$ for all $f \in C_{0}(x), \alpha>0$.

(iv) The family of functions $g$ which are continuous and $\alpha$-supermedian wrt. $U_{\alpha}$ for some $\alpha>0$ (i.e. $g \geqslant \beta U_{\alpha+\beta} g$ for all $\beta>0$ ) separates the points of $\mathfrak{X}$.

In [5] Boboc, Bucur and Cornea discuss Ray processes and establish a close connection between these and $\mathrm{H}$-cones. In view of the results of this paper it is natural to ask for a more direct relation between harmonic morphisms and $\mathrm{H}$-cones.

If $v \geqslant 0$ is superharmonic we define the harmonic carrier by

$$
s_{h}(v)=X \backslash\{x ; v \text { is harmonic in a nbd. of } x\}
$$

and if $v \geqslant 0$ is excessive and locally bounded, we define the fine harmonic carrier of $v$ by

$s_{h}^{f}(v)=X-\{x ; v$ is finely harmonic in a fine nbd. of $x\}$. 
LEMMA 1. $S_{h}(v)$ and $S_{h}^{f}(v)$ are carriers on the non-negative superharmonic functions and the locally bounded excessive functions, respectively, in the sense that
a) ${ }_{h} S_{h}(v)=\phi \Leftrightarrow v$ is harmonic on $X$
b) $v_{1} \leqslant v_{2} \Rightarrow S_{h}\left(v_{1}\right) \subset s_{h}\left(v_{2}\right)$
c) For any $v \geqslant 0$ superharmonic and any two closed subsets $F_{1}, F_{2}$
of $X$ such that $\mathrm{F}_{1} \cup \mathrm{F}_{2}=\mathfrak{X}$ there exist $\mathrm{v}_{1}, \mathrm{v}_{2} \geqslant 0$ superharmonic such that $v=v_{1}+v_{2}, s_{h}\left(v_{i}\right) \subset F_{i}, i=1,2$,

and similarly for $\mathrm{s}_{\mathrm{h}}^{\mathrm{f}}$.

Proof. We give the proof for $S_{h}$, the proof for $S_{h}^{f}$ being similar.

a) This is trivial.

b) Suppose $v_{1}, v_{2}$ are non-negative superharmonic functions such that $v_{1} \lesssim v_{2}$, i.e. there exists a non-negative superharmonic function $\theta$ such that

$$
\mathrm{v}_{2}=\mathrm{v}_{1}+\theta
$$

Suppose $\mathrm{v}_{2}$ is harmonic in an open set $W$. Then for any open set $\mathrm{V} \subset \mathrm{W}$ we have

$$
\mu \mathrm{v}_{2}=\mathrm{v}_{2}
$$

and $\mu \mathrm{v}_{1} \leqslant \mathrm{v}_{1}, \mu \mathrm{V}_{\theta} \leqslant \theta$. Hence

$$
\mathrm{v}_{2}=\mu \mathrm{V}_{2}=\mu \mathrm{v}_{1}+\mu \mathrm{V}_{\theta} \leqslant \mathrm{v}_{1}+\theta=\mathrm{v}_{2} \text {, }
$$

and therefore 


$$
\mu \mathrm{v}_{1}=\mathrm{v}_{1} \text { for all such } \mathrm{v} \text {. }
$$

It follows that $v_{1}$ is harmonic in $w$. This proves that $s_{h}\left(v_{1}\right) \subset s_{h}\left(v_{2}\right)$.

c) Let $v>0$ be superharmonic and $F_{1}, F_{2}$ closed, $F_{1} \cup F_{2}=\nVdash$. Consider the Riesz decomposition of $v$ :

$$
v=p_{0}+h_{0}
$$

There exist potentials $p_{1}, p_{2}$ sit.

$$
p_{0}=p_{1}+p_{2}, s\left(p_{i}\right) \subset F_{i}, i=1,2, \quad([7], p .194)
$$

(In the fine topology case the same result is true, as can be seen by modifying the proof on p. $194 i[7]$, using (2.1).)

Now let $v_{1}=p_{1}, v_{2}=p_{2}+h_{0}$. Then $v=v_{1}+v_{2}$ and $s_{h}\left(v_{1}\right)=$ $s\left(p_{1}\right) \subset F_{1}, s_{h}\left(v_{2}\right)=S\left(p_{2}\right) \subset F_{2}$. That completes the proof of Lemma 1 .

Recall that an abstract carrier $S(v)$ in the sense of [7], p.186 satisfies $b), c)$ above and-instead of a) $h^{-}$

a) $\mathrm{S}(\mathrm{v})=\phi \Leftrightarrow \mathrm{v}=0$.

Thus $S_{h}$ and $S_{h}^{f}$ are not abstract carriers, but condition a) is not essential for our purposes. We may regard $s_{h}$ and $s_{h}^{f}$ as extensions of $S$ to the set of non-negative superharmonic functions and the set of excessive functions, respectively. One can check that Prop. 8.1.1-8.1.4. in [7] remain valid for $s_{h}$ and $s_{h}^{f}$. If $f$ is continuous, resp. finely continuous we define a corresponding specific multiplication $f * u$ wrt. the harmonic, resp. fine harmonic carriers $s_{h}$ and $s_{h}^{f}$ as on p. 189 in [7]. For notational convenience we will use $f * u$ to denote the specific multiplication in 
these cases also, since it will be clear from the context what carrier we have in mind.

LEMMA 2. Let $v, v^{\prime}$ be non-negative, superharmonic functions on $X$. Let $f$ be continuous on $\nVdash$ with compact support $K$ and assume that $v=v^{\prime}$ in a neighbourhood $W$ of $K$. Then

$$
f * v=f * v^{\prime} \quad \text { on } \quad x
$$

and $S_{h}(f * v)=S_{h}\left(f * v^{\prime}\right) \subset \bar{W}$.

Proof. By Prop. 8.1.4. p. 188 in [7] we can find a partition $\left(w_{1}, w_{2}\right)$ of $v$ such that

$$
s_{h}\left(w_{1}\right) \subset \bar{w}, s_{h}\left(w_{2}\right) \subset s_{h}(v) \sim w
$$

and a partition $\left(w_{1}^{\prime}, w_{2}^{\prime}\right)$ of $v^{\prime}$ such that

$$
s_{h}\left(w_{1}^{\prime}\right) \subset \bar{w}, s_{h}\left(w_{2}^{\prime}\right) \subset s_{h}\left(v^{\prime}\right)-w
$$

Since taking finer partitions only improve the approximations $\delta{ }^{\star} f$ and $\delta_{\star} f$ to $f * v, f * v^{\circ}$, we may assume that the partitions considered are finer than both $\left(w_{1}, w_{2}\right)$ and $\left(w_{1}^{\prime}, w_{2}^{\prime}\right)$. For such partitions $\left(\tilde{w}_{i}\right),\left(\tilde{w}_{i}^{\prime}\right)$ we have

$$
\begin{aligned}
& \sum(\sup f) \tilde{w}_{i}=\sum \quad(\sup f) \tilde{w}_{i} \quad \text { and } \\
& i_{h}\left(\tilde{w}_{i}\right)^{i} \quad s_{h}\left(\tilde{w}_{i}\right) \subset \bar{w} \quad s_{h}\left(\tilde{w}_{i}\right) \\
& \sum_{i}(\sup f) \tilde{w}_{i}^{\prime}=\sum_{\tilde{w}_{i}} \quad\left(\sup _{\tilde{w}} f\right) \tilde{w}_{i}^{\prime} \\
& s_{h}\left(\tilde{w}_{i}^{\prime}\right) \quad s_{h}\left(\tilde{w}_{i}^{\prime}\right) \subset \bar{w} \quad s_{h}\left(\tilde{w}_{i}^{\prime}\right)
\end{aligned}
$$


Taking inf over all such partitions the expressions on the right hand sides will approach $f \star(v \mid W)$ and $f \star\left(v^{\prime} \mid W\right)$ which are identical since $v=v^{\prime}$ on $W$.

LEMMA 3. Let $\mathrm{u}>0$ be continuous and superharmonic on $\mathfrak{X}$. Then there exists a (unique) Ray resolvent $\left\{\tilde{U}_{\alpha}\right\}_{\alpha \geqslant 0}$, such that

$$
\tilde{U}_{0}(f)=f \star u \text { for all } f \in C_{C}(x) \text {. }
$$

$x \in S_{h}^{f}(u) \Leftrightarrow\left(\tilde{U}_{0} f\right)(x)>0$ for all finely continuous

$f \geq 0$ s.t. $f=1$ in a fine neighbourhood of $x$.

Proof. Let $p>0$ be a bounded continuous potential on $\mathcal{X}$. Then for each $n$ the function

$$
p_{n}=\min (u, n p)
$$

is a potential on $\mathcal{X}$. Let

$$
\mathrm{w}_{\mathrm{n}}=\{\mathrm{x} ; \mathrm{u}(\mathrm{x})<\mathrm{np}(\mathrm{x})\} .
$$

Then $\mathrm{w}_{\mathrm{n}}$ is open, $\mathrm{w}_{\mathrm{n}} \subseteq \mathrm{w}_{\mathrm{n}+1}$ and

$$
X=\bigcup_{n=1}^{\infty} w_{n}
$$

Let $\left\{U_{\alpha}^{(n)}\right\}_{\alpha \geqslant 0}$ be the resolvent associated with the potential $p_{n}$ ( $[7]$, Prop. 10.2.2). By Lemma 2 we have for $m>n$

So

$$
U_{0}^{(m)}(f)=f \star p_{m}=f \star p_{n}=U_{0}^{(n)}(f) \quad ; f \in C_{C}\left(W_{n}\right)
$$

$$
U_{\alpha}^{(m)}(f)=\sum_{k=0}^{\infty}(-\alpha)^{k}\left(U_{0}^{(m)}\right)^{k+1}(f)=\sum_{k=0}^{\infty}(-\alpha)^{k}\left(U_{0}^{(n)}\right)^{k+1}(f)=U_{\alpha}^{(n)}(f)
$$

for all $f \in C_{C}\left(w_{n}\right), m>n$. 
So we can define $\left\{\tilde{U}_{\alpha}\right\}_{\alpha \geqslant 0}$ on $C_{C}(x)$ by putting

$$
\tilde{U}_{\alpha}(f)=U_{\alpha}^{(n)}(f) \quad \text { if } \operatorname{supp}(f) \subset W_{n} \text {. }
$$

We extend $\tilde{U}_{\alpha}$ in the usual way to functions $g \geqslant 0$ on $\mathfrak{X}$ by

$$
\tilde{\mathrm{U}}_{\alpha}(g)=\lim _{\mathrm{k} \rightarrow \infty} \tilde{\mathrm{U}}_{\alpha}^{\prime}\left(g_{\mathrm{k}}\right)
$$

where $g_{k} \in C_{C}(x)$ for each $k$ and $g_{k} \uparrow g$.

We have to check that $\left\{\tilde{U}_{\alpha}\right\}_{\alpha \geqslant 0}$ is a Ray resolvent: The properties

$$
\alpha \tilde{U}_{\alpha}(1) \leqslant 1
$$

for all $\alpha>0$

and

$$
\tilde{U}_{\alpha}-\tilde{U}_{\beta}=(\beta-\alpha) \tilde{U}_{\alpha} \tilde{U}_{\beta} \quad \text { for all } \alpha, \beta>0
$$

follow from the corresponding properties of $\left\{U_{\alpha}^{(n)}\right\}$. Moreover, from Prop. 10.2.2 in [7] we know that $\mathrm{U}_{\alpha}^{(n)} \mathrm{f}$ is a bounded continuous function, for all $\mathrm{n}$ and all bounded functions $f$ on $\mathcal{X}$. This gives that

$$
\tilde{U}_{\alpha} f \in C_{0}(x)
$$

for all $f \in C_{0}(x)$

Finally, we claim that

the family of functions $g$ which are continuous and $\alpha$-supermedian (wrt. $\tilde{\mathrm{U}}_{\alpha}$ ) for some $\alpha \geqslant 0$ (i.e. $g \geqslant \beta \tilde{U}_{\alpha+\beta} g$ for all $\beta>0)$ separates the points of $X$.

To prove (2.5) note that all continuous, positive hyperharmonic functions $v$ on $\nVdash$ are $\alpha$-supermedian wrt. $\mathrm{U}_{\alpha}^{(\mathrm{k})}$ for $\alpha=0$ and any k ([7], p.250 the argument here does not use that the potential is strict) and these functions separate the points of $\mathscr{X}$ ([7], Prop. 2.3.2). For such functions $v$ we then have 


$$
\beta U_{\beta}^{(k)} v \leqslant v \quad \text { for all } \beta \geqslant 0
$$

Let $v_{k} \geqslant 0$ be a sequence of functions such that $v_{k}=v$ on $w_{k}$ ' $\mathrm{v}_{\mathrm{k}} \in \mathrm{C}_{\mathrm{C}}(\boldsymbol{X})$ and $\mathrm{v}_{\mathrm{k}} \uparrow \mathrm{v}$ on $\boldsymbol{X}$. Then

$$
\beta \tilde{U}_{\beta}(v)=\lim _{k \rightarrow \infty} \sup \beta \tilde{U}_{\beta}\left(v_{k}\right)=\limsup \beta \tilde{U}_{\beta}^{(k)} v \leqslant v,
$$

so these functions are also $\alpha$-supermedian wrt. $\tilde{U}_{\alpha}$ for $\alpha=0$. That proves claim (2.5). It remains to prove $(2.2)$ : Choose $f \geqslant 0$ finely continuous such that $f=1$ in a fine neighbourhood $V$ of $x$. By Lemma 1 we can find superharmonic functions $u_{1}, u_{2} \geqslant 0$ such that $\mathrm{u}=\mathrm{u}_{1}+\mathrm{u}_{2}, \mathrm{~s}_{\mathrm{h}}^{\mathrm{f}}\left(\mathrm{u}_{1}\right) \subset \mathrm{v}$ and $\mathrm{x} \notin \mathrm{s}_{\mathrm{h}}^{\mathrm{f}}\left(\mathrm{u}_{2}\right)$. Then

$$
\left.\left(\tilde{U}_{0} f\right)(x)=(f * u)(x) \geqslant \operatorname{linf}_{S_{h}^{f}\left(u_{1}\right)} f\right) \cdot u_{1}(x)=u_{1}(x) .
$$

If $u_{1}(x)=0$, then $u-u_{2}$ is a non-negative superharmonic function in a fine neighbourhood of $x$ vanishing at $x$, which forces $u-u_{2}$ to be identically zero in this neighbourhood, and hence $x \notin S_{h}^{f}(u)$. Conversely, if $x \notin S_{h}^{f}(u)$, then $u$ is finely harmonic in a fine neighbourhood $W$ of $x$ and then we can find a partition $u=$ $w_{1}+w_{2}$ with $s_{h}^{f}\left(w_{1}\right) \subset w$ and $x \notin s_{h}^{f}\left(w_{2}\right)$. Then both $u$ and $w_{2}-$ and hence $w_{1}$-are finely harmonic in a fine neighbourhood $G$ of $x$ so if $f=0$ outside $G$ we have

$$
\left(\tilde{U}_{0} f\right)(x) \leqslant\left(\sup _{s_{h}^{f}\left(w_{1}\right)} f\right) \cdot w_{1}+\left(\sup _{s_{h}^{f}\left(w_{2}\right)} f\right) \cdot w_{2}=0+0=0 .
$$

That completes the proof of Lemma 3 .

DEFINITION. Let $u>0$ be continuous superharmonic, but not harmoharmonic, on $X$. Then the process $z_{t}=z_{t}^{(u)}$ with resolvent $\left\{\tilde{U}_{\alpha}\right\}$ given by Lemma 3 is called the Ray process associated with u.

In the following $\tilde{\mathrm{P}}^{\mathrm{X}}$ will denote the probability law of $\mathrm{z}_{t}$ starting at $x$, while $\tilde{E}^{X}[f]=\int f d \tilde{P}^{X}$ will denote the corresponding 
expectation. The first exit time from a set $H$ will be denoted by $\tau_{H}$ for any of the processes $x_{t}, z_{t}, Y_{t}$, since it will be clear from the context what process we have in mind. We always interpret $f\left(z_{t}\right)$ as 0 if $t \geqslant \xi$ (the life time of $z_{t}$ ).

LEMMA 4. Let $\mathrm{z}_{t}^{(\mathrm{u})}$ be the Ray process associated to a continuous, superharmonic function $u>0$. Then we have

(2.6) g excessive wrt. $z_{t}^{(u)} \Rightarrow g$ hyperharmonic on $x$

and

$$
\begin{gathered}
g \geqslant 0 \text { hyperharmonic on } X \Rightarrow g(x) \geqslant \tilde{E}^{x}\left[g\left(z_{t}\right)\right] \\
\text { for all } x \in X, t \geqslant 0 .
\end{gathered}
$$

Proof. Let $g \geqslant 0$ be excessive wrt. $z_{t}^{(u)}$. Put $g_{n}=\min (g, n)$. Then for all $n$

$$
\alpha \tilde{U}_{\alpha}\left(g_{n}\right) \leqslant g_{n} \text { and } \lim _{\alpha \rightarrow \infty} \alpha \tilde{U}_{\alpha}\left(g_{n}\right)=g_{n} .
$$

Moreover,

$$
\tilde{U}_{\alpha} g_{n}=\tilde{U}_{0}\left(g_{n}-\alpha \tilde{U}_{\alpha} g_{n}\right)=\left(g_{n}-\alpha \tilde{U}_{\alpha} g_{n}\right) \star u,
$$

so that $\tilde{\mathrm{U}}_{\alpha} \mathrm{g}_{\mathrm{n}}$ is hyperharmonic. Therefore by Prop. 10.1.5 in [7] $\mathrm{g}$ is the limit of an increasing sequence of hyperharmonic functions and we conclude that $g$ is hyperharmonic. This proves $(2.6)$.

To prove $(2.7)$, let $v \geqslant 0$ be hyperharmonic on $X$.

As in the proof of Lemma 3 we let

$$
\mathrm{p}_{\mathrm{n}}=\min (\mathrm{u}, \mathrm{np}), \mathrm{w}_{\mathrm{n}}=\{\mathrm{x} ; \mathrm{u}(\mathrm{x})<\mathrm{n} \mathrm{p}(\mathrm{x})\}
$$

with associated resolvent $\left\{U_{\alpha}^{(n)}\right\}_{\alpha \geqslant 0}$. Applying Prop. 10.2.1 in [7] to the case $\mathrm{f}=\mathrm{v}, \mathrm{g}=\mathrm{U}_{\alpha}^{(\mathrm{n})} \mathrm{v}$ we get

$$
\alpha U_{\alpha}^{(n)} v \leqslant v
$$

Let $\left\{\mathrm{v}_{\mathrm{k}}\right\}$ be an increasing sequence of continuous potentials with compact support such that 


$$
\mathrm{v}=\lim _{\mathrm{k} \rightarrow \infty} \mathrm{v}_{\mathrm{k}}
$$

Then by applying $(2.7)$ to $\mathrm{v}_{\mathrm{k}}$ we obtain

$$
\alpha \tilde{U}_{\alpha} \mathrm{v}=\lim _{\mathrm{k} \rightarrow \infty} \alpha \tilde{U}_{\alpha} \mathrm{v}_{\mathrm{k}}=\lim _{\mathrm{k} \rightarrow \infty} \alpha \mathrm{U}_{\alpha}^{\left(\mathrm{n}_{\mathrm{k}}\right)} \mathrm{v}_{\mathrm{k}} \leqslant \lim _{\mathrm{k} \rightarrow \infty} \mathrm{v}_{\mathrm{k}}=\mathrm{v}
$$

where $n_{k}$ is chosen so large that $w_{n_{k}}$ contains the support of $v_{k}$. By Theorem 3.6 (iii) in [12] (2.9) is equivalent to (2.7). That completes the proof.

THEOREM 1. Let $q>0$ be a bounded continuous strict potential on $y$ with associated process $Y_{t}$, and let $\phi: X \rightarrow Y$ be a continuous function. Then the following are equivalent:

(1) $\phi$ is a harmonic morphism.

(2) There exists a Ray process $z_{t}$ on $\mathcal{X}$ with the following properties

$$
\xi=0 \text { a.s. } \tilde{\mathrm{P}}^{\mathrm{X}} \Rightarrow \phi \text { finely locally constant at } \mathrm{x}
$$

and

$$
\phi \text { is } Z_{t}-Y_{t} \text { pathpreserving (without time change). }
$$

Proof. (2) (2) (1). Assume (2) holds. Suppose $g$ is $Y_{t}$-excessive in an open set $G \subset Y$. Then for all open sets $W \subset \subset G$ and $y \in W$ we have

$$
g(y) \geqslant \widehat{E}^{Y}\left[g\left(Y_{\tau}\right)\right]
$$

and

$$
g(y)=\lim _{t \rightarrow 0} \hat{E}^{Y}\left[g\left(Y_{t \wedge \tau}\right)\right] \quad \text { for all stopping times } \tau \leqslant \tau_{W} \text {. }
$$

So by (iii) we get, if $y=\phi(x)$ and $\xi>0$ a.s. $\tilde{P}^{x}$

$$
g(\phi(x)) \geqslant \tilde{E}^{\mathbf{x}}\left[(g \circ \phi)\left(z_{\tau}\right)\right],
$$

and 


$$
g(\phi(x))=\lim _{t \rightarrow 0} \tilde{E}^{x}\left[(g \circ \phi)\left(z_{t \wedge \tau}\right)\right] \quad \begin{aligned}
& \text { for all stopping } \\
& \text { times } \tau \leqslant \tau_{U^{\prime}} U=\phi^{-1}(W)
\end{aligned}
$$

On the other hand, if $\mathrm{y}=\phi(\mathrm{x})$ and $\xi=0$ a.s. $\tilde{\mathrm{P}}^{\mathrm{X}}$ then by (i) $\phi$ is finely locally constant near $x$ so clearly $g \circ \phi$ is $z_{t}$-excessive near $x$. We conclude that $g \circ \phi$ is $z_{t}$-excessive on $U$. Hence by (ii) go $\phi$ is $U$ - hyperharmonic in $\phi^{-1}(G)$.

$(1) \Rightarrow(2)$ : Assume that $\phi$ is a harmonic morphism. Then the function
(2.10)
$\mathrm{u}=\mathrm{q} \circ \phi$

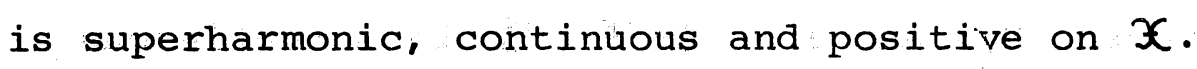

Let $z_{t}=z_{t}^{(u)}$ be the Ray process associated with $u$. Then (ii) holds by Lemma 4 . To prove the remaining part of (2) we first establish that

$$
(f \circ \phi) \star u=(f * q) \circ \phi \text { for all } f \in C^{b}(y):
$$

Consider a partition $\delta=\left(q_{i}\right)_{i \in I}$ of $q([7], p .188)$. Since $\phi$ is a harmonic morphism, we get that $\mu=\left(q_{i} \circ \phi\right)_{i \in I}$ is a partition of $\mathrm{u}=\mathrm{q} \circ \phi$. Moreover, since $\phi$ is harmonic morphism we see that

$$
s_{h}\left(q_{i} \circ \phi\right) \subset \phi^{-1}\left(s q_{i}\right) \quad \text { for all } i
$$

Therefore, using the notation of $[7]$, p.189,

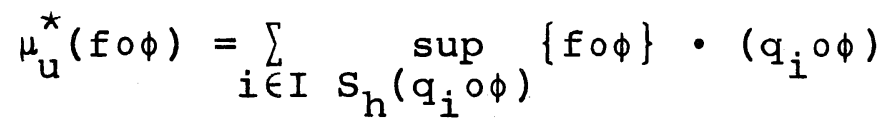

$$
\begin{aligned}
& =\sum_{i \in I} \sup _{\phi\left(s_{h}\left(q_{i} \circ \phi\right)\right)}\{f\} \cdot\left(q_{i} \circ \phi\right)<\sum_{i \in I} \sup _{\operatorname{sq}_{i}}\{f\} \cdot\left(q_{i} \circ \phi\right) \\
& =\left(\sum_{i \in I} \sup _{\operatorname{Sq}_{i}}\{f\} \cdot q_{i}\right) \circ \phi=\delta_{q}^{\star}(f) \circ \phi \text {, for } f \in c^{b}(y) \text {. }
\end{aligned}
$$


Similarly,

$$
\mu_{\star u}(f \circ \phi) \geqslant \delta_{\star q}(f) \circ \phi
$$

So

$$
\delta_{\star q}(f) \circ \phi \leqslant \mu_{\star u}(f \circ \phi) \leqslant \mu_{u}^{\star}(f \circ \phi) \leqslant \delta_{q}^{\star}(f) \circ \phi
$$

and therefore, replacing $\leqslant$ by $\lesssim$ and using the notation from $[6]$, p. 189

(2.13) $\bigvee_{\delta \in \Delta_{q}} \delta_{\star q}(f) \circ \phi \leq \bigvee_{\mu \in \Delta_{u}}^{\mu} \mu_{\star u}(f \circ \phi) \leq \mathcal{\curlywedge}_{\mu \in \Delta_{u}}^{\mu{ }_{u}^{*}}(f \circ \phi) \leq \mathcal{\curlywedge}_{\delta \in \Delta_{q}} \delta_{q}^{\star}(f) \circ \phi$, where $\Delta_{q}$ is the family of all partitions of $q$.

Since the left and right hand side of (2.13) are equal ([7], Prop. 8.1 .5$, p. 189) we conclude that

$(f \star q) \circ \phi=\bigvee_{\delta \in \Delta_{q}} \delta_{\star q}(f) \circ \phi=\bigvee_{\mu \in \Delta_{u}} \mu_{u}^{\star}(f \circ \phi)=(f \circ \phi) \star u$, which is $(2.11)$.

If we introduce the operator

$$
V_{q}(f)(x)=(f \star q)(x), \quad f \in c^{b}(y)
$$

and similarly for $v_{u^{\prime}}$ then $(2.11)$ takes the form

$$
v_{u}(f \circ \phi)=\left(v_{q} f\right) \circ \phi \quad ; f \in C^{b}(y)
$$

From this we conclude that

$$
\tilde{U}_{0}(f \circ \phi)=\hat{U}_{0}(f) \circ \phi \quad \text { for all } f \in C^{b}(y) \text {, }
$$

where $\left\{\tilde{U}_{\alpha}\right\}_{\alpha \geqslant 0}, \quad\left\{\hat{U}_{\alpha}\right\}_{\alpha \geqslant 0}$ are the resolvents of $Z_{t}, Y_{t}$, respectively. Therefore, if $\tilde{A}, \hat{A}$ are the generators of $Z_{t}{ }^{\prime} Y_{t}$, rspectively, we get for $f \in C^{b}(y)$ 


$$
\tilde{A}\left(\hat{U}_{0}(f) \circ \phi\right)=\tilde{A}\left(\tilde{U}_{0}(f \circ \phi)\right)=f \circ \phi=\hat{A}\left(\hat{U}_{0}(f)\right) \circ \phi .
$$

In other words, for all $f \in C^{b}(y)$ we have that if we put $g=\hat{U}_{0}$ ( $f$ ) then $g \in D(\tilde{A}), g \circ \phi \in D(\tilde{A})$ (where $D$ denotes domain of definition) and

$$
\tilde{\mathrm{A}}[\mathrm{g} \circ \phi]=\hat{\mathrm{A}}[\mathrm{g}] \circ \phi
$$

We prove that (2.15) implies that $\phi$ is $Z_{t}-Y_{t}$ path-preserving (without time change) by adopting the argument used to prove Theorem 1 in [8]. (See also [18]). That completes the proof of (2)(iii).

Finally we note that (i) follows from (2.2) of Lemma 3 together with the following 2 statements:

$$
\xi=0 \quad \text { a.s. } \quad \tilde{\mathrm{P}}^{\mathrm{x}} \Leftrightarrow\left(\tilde{\mathrm{U}}_{0} 1\right)(\mathrm{x})=0
$$

$$
x \notin S_{h}^{f}(u) \Leftrightarrow \phi \text { is finely locally constant at } x
$$

The statement $(2.16)$ is clear from the interpretation

$$
\left(\tilde{U}_{0} 1\right)(x)=\int_{0}^{\infty} \tilde{P}^{x}\left(z_{t} \in X\right) d t=\int_{0}^{\infty} \tilde{P}^{x}(t<\xi) d t
$$

To prove (2.17) assume that $u$ is finely harmonic in a relatively compact neighbourhood $U$ of $x$. Then for all $x_{0} \in U$ we have

$$
u\left(x_{0}\right)=E^{x_{0}}\left[u\left(x_{\tau_{U}}\right)\right]=E^{x_{0}}\left[q\left(\phi\left(x_{\tau_{U}}\right)\right)\right]
$$

i.e.

$$
q\left(\phi\left(x_{0}\right)\right)=\int_{\partial U}(q \circ \phi)(z) d \lambda x_{0}(z) ;
$$

where $\lambda_{x_{0}}(F)=E^{x_{0}}\left[x_{\tau_{U}} \in F\right]$ is the harmonic measure of $x_{t}$ w.r.t. $\mathrm{U}$ and $\mathrm{x}_{0}$. In other words, with $\mathrm{y}_{0}=\phi\left(\mathrm{x}_{0}\right)$ and $\eta=\phi(\lambda)$ we have

$$
q\left(y_{0}\right)=\int q d \eta
$$


Since $q$ is strict, this implies that $\eta=\delta_{y_{0}}$ (the point mass at $\left.\mathrm{y}_{0}\right)$. Since this holds for all $\mathrm{x}_{0} \in U$ we conclude that $\phi$ must be constant in U. That completes the proof of Theorem 1 .

Note that $z_{t}$ exits from compact sets $K$ in a finite time a.s., since $Y_{t}$ exits from the compact $\phi(K)$ in a finite time. Also the exit time $\tau$ from $\mathcal{X}$ of $z_{t}$ (i.e. the life time of $z_{t}$ ) need not be infinite. For example, if $\phi(x)$ has a compact closure in $\mathcal{Y}$, we know that $\tau<\infty$ a.s. $\tilde{\mathrm{P}}^{\mathrm{X}}$ (see prop.9).

REMARK. It is natural to ask if it is also true that a harmonic morphism $\phi$ must be $X_{t}-Y_{t}$ path-preserving, in the sense that $\phi$ maps a time change of $X_{t}$ into $Y_{t}$. However, the following example due to Cornea (described in [11]) shows that this need not be the case, at least if we require the time change to be continuous:

Let $X=([-1,1] \times\{0\}) \cup(\{0\} \times[0,1]) \subset \mathbb{R}^{2}$, equipped with the sheaf of "harmonic" functions $h$ which are locally affine outside $(0,0)$ and satisfying the condition that the sum of the 3 one-sided derivatives at $(0,0)$ is zero, i.e. $h(\Delta x, 0)+h(-\Delta x, 0)+h(0, \Delta y)=$ $3 h(0,0)$.

Let $Y=[-1,1]$, equipped with the sheaf of locally affine functions. Then the projection $\phi(x, y)=x$ is a harmonic morphism from $\mathcal{X}$ to y. The process $z_{t}$ in this case will have $B=\{0\} \times(0,1]$ as its branch set, while $Z_{t}$ behaves like $Y_{t}$ (which is a time change of Brownian motion) on $[-1,1] \times\{0\}$. The process $x_{t}$ is a time change of (linear) Brownian motion outside $(0,0)$, and starting from $(0,0)$ its laws on the 3 segments $[-1,0] \times\{0\},[0,1] \times\{0\}$ and $\{0\} \times[0,1]$ are identical. 


\section{§3. A DESCRIPTION OF THE BRANCH SET}

From now on we let $\phi$ denote a non-constant harmonic morphism from $\mathcal{F}$ to $Y$ and let $z_{t}$ be its corresponding Ray process. Recall that the branch set $B$ of $z_{t}$ is defined by

(3.1) $\quad \mathrm{B}=\mathfrak{X}-\left\{\mathrm{x} ; \alpha \tilde{U}_{\alpha} f(x) \rightarrow f(x)\right.$ as $\alpha \rightarrow \infty$, for all $\left.f \in C^{b}(x)\right\}$

$B$ is a Borel set and $B$ is a polar set for $Z_{t^{\prime}}$ i.e.

$$
\tilde{P}^{x}\left[\exists t \geqslant 0, z_{t} \in B\right]=0 \quad \text { for all } x \in X
$$

(see $[13], p .9)$. Note that, for $f \in C_{0}(x)$

$$
\begin{gathered}
\lim _{\alpha \rightarrow \infty} \alpha \tilde{U}_{\alpha} f(x)=\lim _{\alpha \rightarrow \infty} \alpha \tilde{E}^{x}\left[\int_{0}^{\infty} e^{-\alpha t} f\left(z_{t}\right) d t\right] \\
=\lim _{\alpha \rightarrow \infty} \tilde{E}^{x}\left[\int_{0}^{\infty} e^{-s} f\left(z_{\frac{s}{\alpha}}\right) d s\right]=\tilde{E}^{x}\left[f\left(z_{0}\right)\right],
\end{gathered}
$$

and therefore

$$
\mathrm{B}=\left\{\mathrm{x} ; \mathrm{z}_{0}=\mathrm{x} \text { a.s. } \tilde{\mathrm{P}}^{\mathrm{x}}\right\}
$$

In view of Theorem 1 it is natural to investigate the connection between $\phi, B, z_{t}$ and $x_{t}$. Some such results will now be established. Our main result in this section is that $B$ consists of those points $x$ such that $\phi$ is finely locally constant near $x$ (Theorem $2)$. To obtain this we first prove some auxiliary results:

LEMMA 5. Suppose $\phi$ is finely locally non-constant at $x$. Then $x \notin B$.

Proof. Let $G$ be an open neighbourhood of $x$. Put $\phi_{1}=\phi \mid G$. Then $\phi_{1}$ is a harmonic morphism, so by (2.11) we have

$$
\left(f \circ \phi_{1}\right) \star u_{1}=(f * q) \circ \phi_{1} \quad \text { for all } f \in c^{b}(y) \text {, }
$$


where as before $q>0$ is the potential on $\mathcal{Y}$ from which $Y_{t}$ is constructed and $u_{1}=q \circ \phi_{1}$. Therefore, if $H_{t}$ denotes the Ray process associated to $u_{1}$ with probability law $Q^{X}$, then $\phi_{1}$ is $\mathrm{H}_{t}-\mathrm{Y}_{t}$ pathpreserving without time change.

Now note that for $g \in C_{0}(G), z \in G$ we have

$$
U_{0}^{(1)} g(z)=\left(g * u_{1}\right)(z)=(g \star u)(z)=\tilde{U}_{0} g(z),
$$

where $\left\{U_{\alpha}^{(1)}\right\}_{\alpha \geqslant 0,}\left\{\tilde{U}_{\alpha}\right\}_{\alpha \geqslant 0}$ are the resolvents of $H_{t}$ and $z_{t}$. respectively. Hence

$$
\mathrm{U}_{\alpha}^{(1)} \mathrm{g}=\tilde{\mathrm{U}}_{\alpha} \mathrm{g} \text { in } \mathrm{G} \text {, }
$$

for all $\alpha \geqslant 0$ and all $g \in C_{0}(G)$.

It follows by the construction of the processes $H_{t}$ and $z_{t}$ from $\left\{U_{\alpha}^{(1)}\right\}$ and $\left\{\tilde{U}_{\alpha}\right\} \quad$ (see [13], p.10) that for each compact $K \subset G$ the processes ${ }_{t \wedge \tau_{K}}$ and ${ }_{t \wedge \tau_{K}}$ are identical in law.

In particular, since $\phi$ is finely locally non-constant at $x$ we have $\xi>0$ a.s. $Q^{x}$ by Theorem $1,(2)(i)$. Hence $H_{0} \in G$ a.s. and therefore

$$
z_{0} \in G \quad a \cdot s
$$

Since $G$ was an arbitrary neighbourhood of $x$ : we conclude that

$$
\mathrm{z}_{0}=\mathrm{x} \quad \mathrm{a} \cdot \mathrm{s}
$$

and hence $\mathbf{x} \notin B$, as claimed.

In general the $\mathrm{z}_{t}$-excessive functions need not constitute all the non-negative hyperharmonic functions on $\mathfrak{X}$. However, the next result shows that this is the case outside the branch set B: 
LEMMA 6 Let $v \geqslant 0$ be $U$-hyperharmonic on $X$. Then $v$ is $z_{t}-$ excessive on $\mathfrak{X}-B$, in fact

$$
E^{x}\left[v\left(z_{t}\right)\right] \leqslant v(x) \quad \text { for all } t \geqslant 0, x \in X
$$

and

$$
\lim _{t \rightarrow 0} E^{x}\left[v\left(z_{t}\right)\right]=v(x) \quad \text { for all } x \in \mathcal{X}-B
$$

Proof. We have already proved the first part in Lemma $4,(2.6)$. To prove the last part choose $x \in \mathfrak{X}-\mathrm{B}$.

Then

$$
\lim _{\alpha \rightarrow \infty} \alpha \tilde{U}_{\alpha} f(x)=f(x) \quad \text { for all } f \in C^{b}(x) \text {. }
$$

Therefore, with $\mathrm{v}_{\mathrm{k}}$ as in Lemma 4,

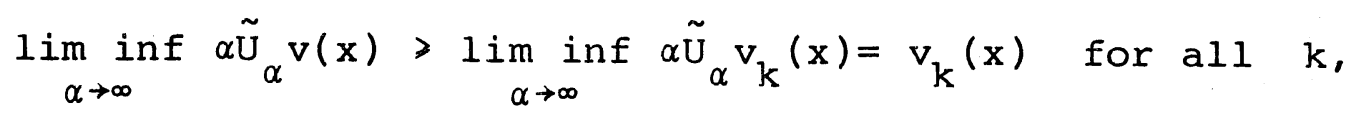

and hence

$$
\lim _{\alpha \rightarrow \infty} \alpha \tilde{U}_{\alpha} v(x)=v(x)
$$

which is equivalent to (3.7).

It is natural to ask for a connection between the $x_{t}$-finely open and the $\mathrm{z}_{t}$-finely open sets. For a given Borel set $\mathrm{H}$ in $\mathfrak{X}$ we define

$$
\mathrm{B}_{\mathrm{H}}=\left\{\mathrm{x} \in \mathrm{B} ; \mathrm{Z}_{0} \in \mathrm{H} \quad \text { a.s. } \quad \tilde{\mathrm{P}}^{\mathrm{x}}\right\}
$$

Then we have:

\section{LEMMA 7}

a) Suppose $U$ is $x_{t}$-finely open. Then $U \backslash B$ and $(U \backslash B) U \cdot B_{U}$ are $\mathrm{z}_{t}$-finely open.

b) Suppose $V$ is $z_{t}$-finely open. Then $V U B_{V}$ is $x_{t}$-finely open. 


\section{Proof.}

a) Suppose $U \subset X$ is $x_{t}$-finely open. Define $f(x)=E^{x}\left[\tau_{U}\right]$. Then $f$ is $x_{t}$-excessive and $f(x)>0 \Leftrightarrow x \in U$. By Lemma 61 we get that

$$
0<f(x)=\lim _{t \rightarrow 0} \tilde{E}^{x}\left[f\left(z_{t}\right)\right] \text { for } x \in U \backslash B
$$

Hence $z_{t}$ stays in $U \backslash B$ for a positive period of time a.s. when starting from a point in $U \backslash B$. It follows that $U \backslash B$ and so $(U \backslash B) \cup B_{U \backslash B}=(U \backslash B) \cup B_{U}$ is $z_{t}$-finely open.

b) Suppose $V$ is $Z_{t}$-finely open.

Define $g(x)=\tilde{E}^{x}\left[\tau_{v}\right]$. Then $g$ is . $z_{t}$-excessive and $\mathrm{g}(\mathrm{x})>0 \Leftrightarrow \mathrm{x} \in \mathrm{V} \cup \mathrm{B}_{\mathrm{V}}$. By Lemma $4 \mathrm{~g}$ is $\mathrm{x}_{\mathrm{t}}$-excessive, so $\mathrm{VUB}_{\mathrm{V}}=\{\mathrm{x} ; \mathrm{g}(\mathrm{x})>0\}$ is $\mathrm{x}_{\mathrm{t}}$-finely open.

LEMMA 8. Suppose $U \subset X$ is $x_{t}$-finely open. Then $U \backslash B$ is $z_{t^{-}}$ finely open and $\phi(U, B)$ is $Y_{t}$-finely open.

Proof. If $U$ is $x_{t}$-finely open, it follows from Lemma 7 that $U \backslash B$ is $z_{t}$-finely open. Hence $z_{t}$ stays in $U \cdot B$ for a positive period of time a.s. when starting from a point in UVB. It follows that $\phi\left(z_{t}\right)$ stays in $\phi(U \backslash B)$ for a positive period of time a.s. when starting from $\phi(x) \in \phi(U \backslash B)$. By Theorem $1 Y_{t}$ has the same law as $\phi\left(z_{t}\right)$, so the set $\phi(U \backslash B)$ must be finely open.

Adding up these results we obtain: 
THEOREM 2. The following are equivalent:

1) $\phi$ is finely locally constant at $x$

2) There exists a fine neighbourhood U of $x$ such that $\phi(x) \notin \phi(U) '$, where ' denotes $\mathrm{Y}_{t}$-fine interior.

3) $x \in B$

Proof: 1) $\Rightarrow$ 2) is trivial.

2) $\Rightarrow 3$ ) follows from Lemma 8: If $x \notin B$ and $U$ is a fine neighbourhood of $x$ then $\phi(U)^{\prime} \supset \phi(U \backslash B)^{\prime}=\phi(U \backslash B) \neq \varnothing$.

$3) \Rightarrow 1$ ) follows from Lemma 5 .

A fundamental result due to Constantinescu \& Cornea [6, Theorem 3.5] says that every non-constant harmonic morphism between Brelot harmonic spaces $X$ and $Y$ is finely open, provided that $X$ is connected and the points of $\mathcal{Y}$ are polar. Subsequently it was proved by Fuglede ( $[11]$, Corollary p. 190) that if we put

$$
F=\left\{x_{i} x \text { is not finely isolated in } \phi^{-1}(\phi(x))\right\}
$$

then $\phi$ is finely open outside F. From Theorem 2 we obtain the following complete descripton of when (or where) a harmonic morphism is finely open:

COROLLARY 1. For each $\mathrm{x} \in \mathfrak{X}$ either $\phi$ is finely locally constant at $x$ or else $\phi$ maps every fine neighbourhood of $x$ onto a fine neighbourhood of $\phi(x)$.

Note that $B \subset F$, so Corollay 1 contains Fuglede's result. If $\mathfrak{X}, \mathbb{Y}$ are Brelot harmonic spaces and the points $y \in \mathcal{Y}$ are polar, then $\phi^{-1}(y)$ are polar sets in $X([6$, Theorem 3.2]) and therefore $\phi$ cannot be finely locally constant. Therefore Corollary 1 contains the result of Constantinescu \& Cornea as well. 
Using Theorem 2 and Lemma 6 we can obtain the following partial strengthening of Theorem 1:

THEOREM 3. Let $\psi: X+Y$ be a continuous, finely locally non-constant function.

Then the following are equivalent:

(i) $\psi$ is a harmonic morphism

(ii) $\psi$ is $\mathrm{X}_{t}^{-Y_{t}}$ pathpreserving, with a continuous time change.

Proof. $(i i)=)(i)$ : The proof that $(i i) \Rightarrow(i)$ in Theorem 1 applies. $(i)=>(i i)$ : Suppose $\psi$ is a harmonic morphism and let $z_{t} b$ the Ray process with branch set B associated to $\psi$ by Theorem 1 . Since $\psi$ is locally non-constant we have $B=\varnothing$, by Theorem 2. From Lemma 6 we conclude that $z_{t}$ and $x_{t}$ have the same excessive functions. Hence $z_{t}$ and $x_{t}$ have the same hitting distributions, by Theorem 8.1 in Blumenthal, Getoor and McKean [3]. (Both $x_{t}$ and $z_{t}$ satisfy Hunt's conditions (C) and (E), see [14] p.89 and p.330). Therefore $z_{t}$ is a continuous time change of $x_{t}$, by the main result in $[3]$.

\$4. OTHER APPLICATIONS.

We now turn to the question of polar sets. First we relate the polar sets of $x_{t}$ to those of $z_{t}$ :

LEMMA 9. $x_{t}$ and $z_{t}$ have the same hitting distributions outside $B$, i.e. for any compact set $K \subset K-B$ and any bounded continuous function $f$ on $K$ and all $x \in X-B$ we have

$$
\mathrm{E}^{\mathrm{X}}\left[\mathrm{f}\left(\mathrm{X} \tau_{\tau_{K}}\right) ; \tau_{K}<\infty\right]=\tilde{E}^{\mathrm{X}}\left[\mathrm{f}^{\prime}\left(\mathrm{z}_{\tau_{K}}\right) ; \tau_{K}<\infty\right]
$$


Proof. Since both $x_{t}$ and $z_{t}$ satisfy Hunt's conditions (C) and (E) ( $[14]$, p.89 and p.330) this follows from Theorem 8.1 in Blumenthal, Getoor and McKean [3].

Fuglede ([11], Theorem 1.3) has proved that if $\phi$ is locally nonconstant, then $\phi^{-1}(F)$ is polar if $F$ is polar. Without the assumption that $\phi$ is locally non-constant we obtain the following:

THEOREM 4. Let $H$ be a polar set in $y$. Then $\phi^{-1}(H) \backslash B$ is a polar set in $X$.

Proof. It is clear that $\phi^{-1}(\mathrm{H})$ is polar for $\mathrm{Z}_{t^{\prime}}$ by Theorem 1 . Since $x_{t}$ and $z_{t}$ have the same polar sets outside B (Lemma 9), the result follows.

Finally we mention the following boundary value result, which follows from Theorem 1 and the fact that the paths of $Y_{t}$ are continuous, by adopting the proof of Theorem 2, p. 232-234 in [8]:

THEOREM 5. (Boundary value theorem for harmonic morphisms) Let Vс $\mathfrak{X}$ be open, $\overline{\mathrm{V}}$ compact. Let $\phi: V \rightarrow Y$ be a finely locally non-constant harmonic morphism

Then

$$
\lim _{t \rightarrow \tau_{V}} \phi\left(x_{t}\right) \quad \text { exists a.s. } P^{X} \text {, for all } x \in V \text {. }
$$

Proof. By Theorem 3, we conclude that

$$
\lim _{t \rightarrow \tau_{V}} \phi\left(x_{t}\right)=\lim _{t \rightarrow \tau_{V}} \phi\left(H_{t}\right)=\lim _{t \rightarrow \tau_{V}} K_{t}=\lim _{t \rightarrow \tau_{V}} Y_{t} \quad a \cdot s \cdot
$$

and the last limit exists a.s. because $\phi(\bar{v})$ is compact.

If $V \subset X$ is open and $f: V \rightarrow \mathcal{Y}$ we say that $f$ has an asymptotic value $w$ at $y \in \partial V$ if there exists a curve $\gamma$ in $V$ terminating at $y$ such that 


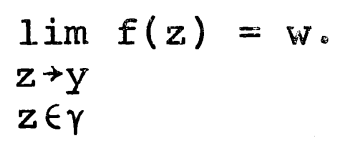

The harmonic measure $\lambda=\lambda_{\mathrm{a}, \mathrm{V}}^{\mathrm{X}}$ associated to the process $\mathrm{x}_{t}$ for the set $V$ is defined by

$$
\lambda_{\mathrm{a}}(\mathrm{H})=\mathrm{P}^{\mathrm{a}}\left[\mathrm{Z}_{\tau_{\mathrm{V}}} \in \mathrm{H} ; \tau_{\mathrm{V}}<\infty\right]
$$

With these concepts we can formulate the following immediate consequence of Theorem 5:

THEOREM 6. (Asymptotic value theorem). Let $\phi$ and V satisfy the conditions of Theorem 5. Then $\phi$ has an asymptotic value at a.a. boundary points $y \in \partial V$ w.r.t. the harmonic measure for $x_{t}$.

\section{A problem.}

The following question is natural in view of Theorems 1-3: Define

and

$$
\lambda(x)= \begin{cases}1 ; & x \notin B \\ 0 ; & x \in B, \\ \beta_{t} & =\int_{0}^{t} \lambda\left(x_{s}\right) d s, \alpha_{t}=\inf \left\{s>0 ; \beta_{t}>s\right\}\end{cases}
$$

Is $\phi \mathrm{X}_{\alpha_{t}}-\mathrm{Y}_{t}$ path-preserving?

What is the relation between $\mathrm{x}_{\alpha_{t}}$ and $\mathrm{z}_{t}$ ?

Acknowledgements. We wish to thank A. Cornea and G. Michaletzky for their comments to a preliminary version of this paper. And we are very grateful to B. Fuglede for useful communications throughout this work. 


\section{BIBLIOGRAPHY}

[1] A. Bernard, E.A. Campbell and A.M. Davie: Brownian motion and generalized analytic and inner functions. Ann. Inst. Fourier 29 (1979), 207-228.

[2] R.M. Blumenthal and R.K. Getoor: Markov Processes and Potential Theory. Academic Press 1968.

[3] R.M. Blumenthal, R.K. Getoor and H.P. McKean: Markov processes with identical hitting distributions. Illinois J. Math. $6(1962), 402-420$ (and 7(1963), 540-542).

[4] J. Bliedtner and W. Hansen: Potential Theory. Universitext, Springer-Verlag 1986.

[5] N. Boboc, G. Bucur and A. Cornea: Order and Convexity in Potential Theory: H-Cones. Springer LNM 853 (1981)

[6] C. Constantinescu and A. Cornea: Compactifications of harmonic spaces. Nagoya Math. J. 25(1965), 1-57.

[7] C. Constantinescu and A. Cornea: Potential Theory on Harmonic Spaces. Springer-Verlag 1972.

[8] L. Csink and B. Øksendal: Stochastic harmonic morphisms: Functions mapping the paths of one diffusion into the paths of another. Ann. Inst. Fourier 33 (1983), 219-240.

[9] E.B. Dynkin: Markov Processes II. Springer-Verlag 1965.

[10] B. Fuglede: Harmonic morphisms between Riemannian manifolds. Ann. Inst. Fourier 28 (1978), 107-144.

[11] B. Fuglede: Invariant characterizations of the fine topology in potential theory. Math.Ann. 241 (1979), 187-192.

[12] B. Fuglede: Harnack sets and openness of harmonic morphisms. Math. Ann. 241 (1979), 181-186. 
[13] R.K. Getoor: Markov Processes: Ray Processes and Right Processes. Springer LNM 440 (1975).

[14] G.A. Hunt: Markov processes and potentials I, II. Illiniois J. Math. 1 (1957), 44-93 and 316-369.

[15] I. Laine: Covering properties of harmonic B1-mapping. Ann. Acad. Sci. Fenn. Ser. A.I., Vol. 1 (1975), 309-325.

[16] H.P. McKean: Stochastic Integrals. Academic Press 1969.

[17] B. Øksendal: Finely harmonic morphisms, Brownian path preserving functions and conformal martingales. Inventiones math. 75(1984), 179-187.

[18] B. Øksendal: Stochastic processes, infinitesimal generators and function theory. In S.C. Power (editor): Operators and Function Theory. Reidel 1985.

\author{
Laszlo Csink \\ Faculty of Arts \\ Eötvös University \\ P.O.Box 107 \\ $\mathrm{H}-1364$ Budapest (Hungary)
}

\author{
Bernt $\varnothing \mathrm{ksendal}$ \\ Department of Mathematics \\ University of Oslo \\ P.O.Box 1053 Blindern \\ $\mathrm{N}-0316$ OSLO 3 (Norway)
}


